proportion of time spent on research, number of hours spent weekly on research, proportion of time spent on teaching, class of undergraduate degrec, chronological age, professional age? What is the relationship between recognition and these variables and what is the relationship between productivity and recognition? Competition is measured by the number of times scientists have been anticipated through having research findings similar to their own published first by someone else. What anticipation have British HEP workers experienced, for example in relation to their rank or by comparison with American scientists? Having been anticipated, did they publish or not publish? As for communication, what is the most important method of obtaining information relevant to research, by type of HEP, rank, professional age?
How does verbal communication work and which are the most important journals?

Equally, if not more, illuminating are the extracts from transcripts of conversations about competition. One suggestion is that competition helps to motivate scientists who otherwise might put forward less than their best effort. An experimentalist is quoted as saying, "I have suspicions that less than one physicist in three that I've met is really and deeply and drastically motivated by a desire to "find out'". There is much material in this book to show just how cut-throat the competition can become - both between individuals and between continents-together with material which suggests why American physicists 'beat' European physicists in certain races of discovery. Gaston records his evidence about the "three main methods of deviance" which scientists sometimes use to get the recognition which eludes them: hasty publication; fraud and theft; and secrecy.

In his conclusions, the author does not ask squarely where his own cvidence leaves Merton's four rules of science. He finds that the reward system in British HEP communities, unlike the system in America, operates in a universalistic fashion. Recognition is highly related to productivity and theorists receive more recognition than experimentalists. But affiliation with a particular type of university or a department in a particular prestige category has no effect on opportunities for scientific recognition. The four rules are, however, meant to hang together and Gaston shows that scientists are indeed human in their quest.

Margaret Gowing
The Competent Infant: Research and Commentary. Edited by Joseph L. Stone, Henrietta T. Smith and Lois B. Murphy. Pp. viii $+1,314$. (Tavistock : London, 1974.) $£ 11.00$.

Most books consisting of collections of published papers are worth neither reviewing, buying nor reading: the present volume is an exception. The editors have done a remarkable job of arranging the selected papers in logical order, shortening them, and with the help of their own commentaries producing a coherent book. There are two further reasons why this collection is particularly useful. First, infant development is a topic on which spectacular progress has recently been made and second, as there are few journals devoted specifically to it, papers on the subject have appeared in such a wide range of journals that no one person could possibly scan them all: in The Competent Infant, we find papers from the Journal of Ophthalmology rubbing shoulders with extracts from Psychosomatic Medicine, Vita Humana, and Cognitive Psychology.

The book demonstrates the ways in which our ideas about the competence of infants have been revised drastically by the work of such pioneers as Wolff, Escalona and Prechtl. Until some 20 years ago, it was widely accepted that new-born babies were almost entirely passive and incapable of anything much in the way of organised adaptive behaviour. More recent research shows quite the contrary and the watershed may be placed at the publication of Wolff's now classic paper in 1959. He found that, if you catch your infant at the right moment, it will emit highly organised and complex behaviour. As Prechtl puts it: "If you put a newborn infant baby in a supine position in its cot and cover it with a blanket up to the neck, of course it gives the impression of being a kind of vegetable which just cries and sucks from time to time and that's all'. What Wolff discovered was that the reactivity of the new born depends on its state. He classified such states into sleep (regular, periodic and irregular), drowsiness, alert acvtivity and alert inactivity. It is only when the infant is alert and

\section{More than vegetables}

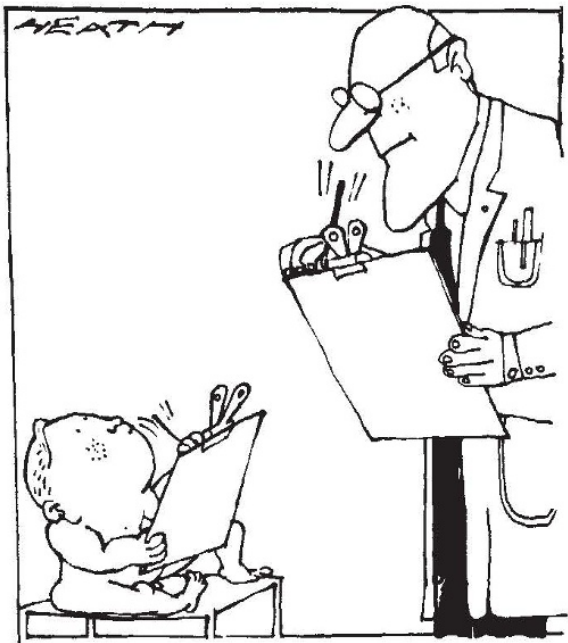

inactive that it will attend to outside stimuli; when it is alert and active, crying or threshing around, it pays little attention to external stimuli, particularly to new stimuli. During the first week of life, the infant spends only about $10 \%$ of the day time in alert inactivity. In this state "his respirations are regular . . . his eyes are wide open, shiny and capable of conjugate eye movements, and he makes visual and auditory pursuit movements ... the skin is pink but not flushed". Such behaviour tends to occur immediately after the baby has satisfied its physiological needs such as feeding and defecating. Experimenters patient enough to wait for such periods have been rewarded by observing complex behaviour even on the first day of life: some infants in fact show about an hour of alert inactivity starting 15 minutes after birth.

Recent research, stemming from Wolff's insights, has shown that there is considerable innate organisation of perceptual and motor functions. One of the most remarkable experiments republished in the present book is Sackett's ingenious demonstration that monkeys have an innate capacity to recognise not merely other monkeys but whether or not the other monkey is in a threat posture; this work is now ncarly 10 years old and its implications are so important that it is very regrettable that, as far as I know, there have been no independent attempts to replicate it. There is also ample evidence that personality traits which tend to remain constant throughout life may appear in new-born infants: American babies of Chinese origin show less irritability and impatience than do whites born on the same ward and tested within a few hours of birth.

The book should give pause to those who think either that psychologists rarely discover anything we did not know already or that introspection and every day experiece are all we need use to infer the structure of the minda belief that has recently been resuscitated by some polemicists for the new branch of technology known as 'artificial intelligence'

The book is 1,300 pages long and nicely produced: at $£ 11.00$ it surely rates as a best buy. 